\title{
Originalia
}

Effektivitätsstudie zum Vergleich einer einmaligen kurzzeitigen manuellen Traktion am kraniomandibulären Gelenk mit einer Placebobehandlung - Qualität Manueller Therapie bei unspezifischen Rückenschmerzen und Therapiemöglichkeiten bei okulären Dysfunktionen sind die Themen dieser Ausgabe. Reichen auch Sie Artikel ein - wir freuen uns darüber!

\section{Einmalige manuelle Traktion bei Patienten mit kraniomandibulären Dysfunktionen}

\author{
Randomisierte kontrollierte Studie
}

\section{One-Off Manual Traction in Patients with Craniomandibular Dysfunctions}

Randomised Controlled Trial

\section{A.-M. Schmitz ${ }^{1}$ H. von Piekartz ${ }^{2}$}

${ }^{1}$ medicos.osnabrück GmbH, Gesundheits- und Rehabilitationszentrum, Osnabrück | ${ }^{2}$ Hochschule Osnabrück, University of Applied Science, Osnabrück eingereicht: 10.5 .2011 | akzeptiert: 3.8.2011

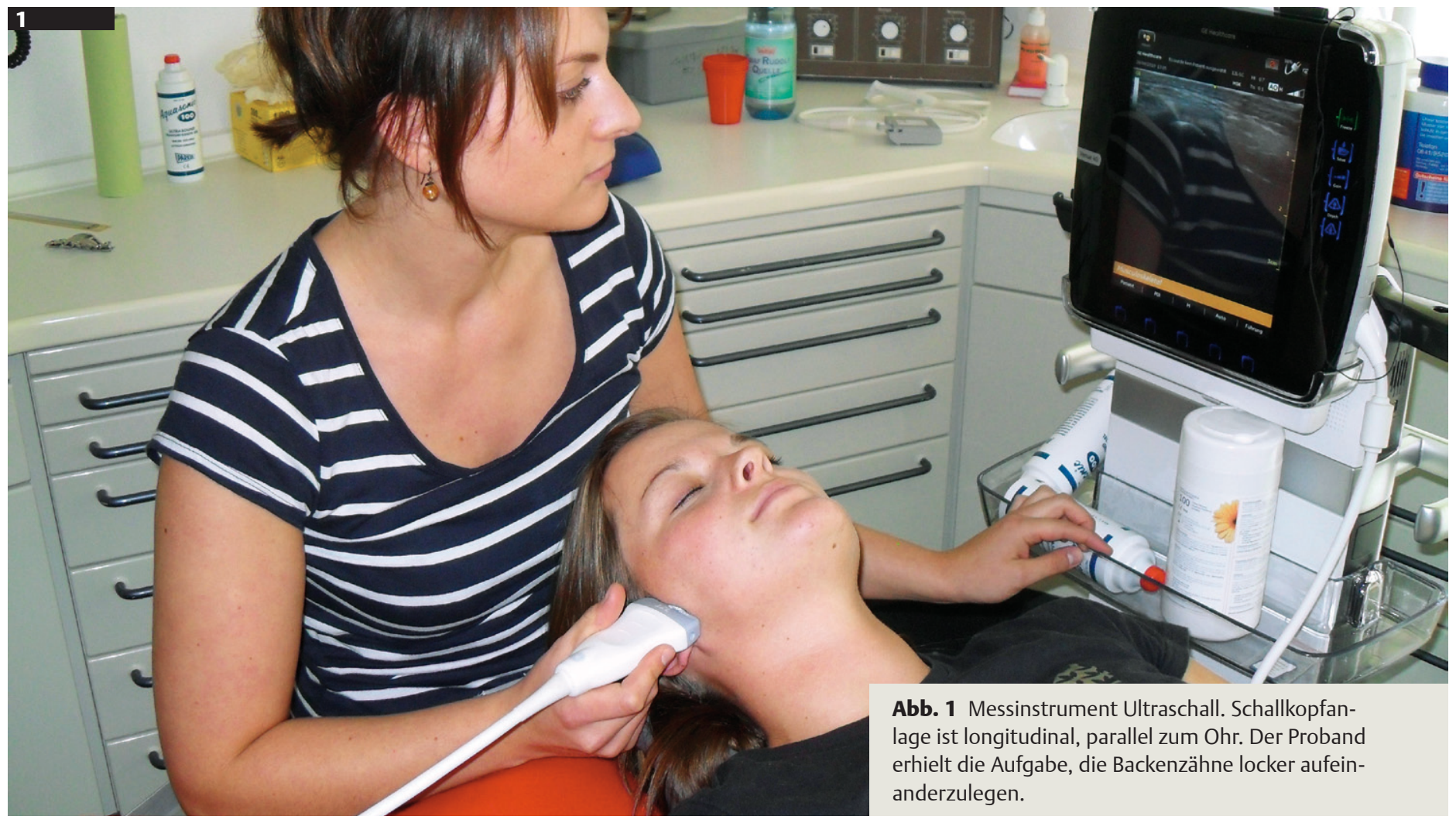




\section{Zusammenfassung}

Passive manuelle Mobilisationen - vor allem die manuelle longitudinale Bewegung nach kaudal (Traktion) - sind in der kraniomandibulären Region eine häufig angewandte wirksame manuelle Intervention.

In dieser randomisierten kontrollierten Studie wurden eine einmalige kurzzeitige manuelle Traktion nach kaudal appliziert und der Einfluss auf den Kiefergelenkspalt bei Patienten mit kraniomandibulären Dysfunktionen (CMD) gemessen. Primär wurde die Distanzveränderung zwischen dem Condylus mandibulae und der Fossa articularis mittels Ultraschall untersucht. Die Messungen nahm eine verblindete Therapeutin direkt nach und 2-4 Tage nach der Behandlung vor. Eine Verblindung aller Probanden war gegeben.

Die Interventionsgruppe wies direkt nach der Behandlung keine statistisch signifikante Veränderung des kraniomandibulären Gelenkspalts auf. Nach 2-4 Tagen zeigte sich eine statistisch signifikante Vergrößerung der Distanz. Zusätzlich gemessene Schmerzen präsentierten keine statistisch signifikanten Unterschiede. Innerhalb der Placebogruppe sowie zwischen beiden Gruppen ergaben sich im Vorher-nachher-Vergleich keine statistisch signifikanten Unterschiede in allen Parametern.

Obwohl die subjektiven Beschwerden beider Gruppen unverändert waren, erzielte die Applikation der einmaligen kurzzeitigen manuellen Traktion von 4 Minuten in der Interventionsgruppe nach 2-4 Tagen eine minimale Veränderung der Distanz des Gelenkspalts. Nach Anwendung der Placebobehandlung ließen sich hingegen keine signifikanten Veränderungen nachweisen.

Gelenkspaltseparation hängt nicht direkt mit der subjektiven Verbesserung der Interventions- und Placebogruppe zusammen.

\section{Schlüsselwörter}

kraniomandibuläre Dysfunktion | manuelle

Gelenkmobilisation | manuelle Traktion |

Effektivität | Ultraschall | Sonografie | Randomisierung

\section{Abstract}

Passive manual mobilisations - predominantly manual longitudinal caudal movement (traction) - are often effectively used as manual therapy intervention in the craniomandibular region.

In this randomised controlled trial a short one-off manual caudal traction was applied and the influence on the temporomandibuar joint gap was measured in patients with craniomandibular dysfunctions (CMD). Primarily change in distance between the mandibular condyle and articular fossa was investigated using ultrasound. A blinded therapist performed the measurements directly after and 2-4 days after treatment. All subjects were also blinded. The intervention group revealed no statistically significant alteration of the temporomandibuar joint gap directly after treatment. After 2-4 days a statistically significant increase in distance became apparent. There were no statistically significant differences in the pain measurements. In the before-after comparison there were no statistically significant differences in all parameters neither within the placebo group nor between the two groups.

Although subjective complaints in both groups remained unchanged, the 4 minute, one-off application of manual traction produced a minimally altered distance in the joint gap in the intervention group 2-4 days after intervention. However no significant changes were seen after a placebo treatment.

Joint gap separation is not closely related to subjective improvement in both the intervention and placebo group.

\section{Key words}

craniomandibular dysfunction | manual joint

mobilisation | manual traction | effectiveness |

ultrasound | sonography | randomisation

\section{Einleitung}

Die kraniomandibulären Dysfunktionen (CMD) umfassen eine Gruppe von Dysfunktionen des Kiefergelenks und der Kaumuskulatur, die einzeln oder kombiniert auftreten [40]. Vermutlich können mehrere Faktoren CMD hervorrufen, die sich gegenseitig beeinflussen [29, 31]. Die häufigsten Symptome der CMD sind Schmerzen im Kiefergelenk-, Kopf- oder Gesichtsbereich und in anderen Körperregionen. Zudem kommen Kiefergelenkgeräusche wie Knacken oder Knirschen, aber auch eine eingeschränkte Beweglichkeit wie Mundöffnungsschwierigkeiten vor. CMD verursachen auch eine schmerzhafte, druckempfindliche Kaumuskulatur und Ohrgeräusche wie Tinnitus oder Rauschen [30, 31].

Etwa $40 \%$ der Patienten mit Schmerzen aufgrund von CMD sind in ihrer Fähigkeit zu arbeiten, an Schule oder Ausbildung teilzunehmen sowie in der Ausübung alltäglicher Aktivitäten einge-

schränkt [18]. Laut einem Review leiden 20\% der Bevölkerung unter CMD, von denen sich 10-20\% in therapeutischer Behandlung befinden [24], was den Behandlungsbedarf dieser Dysfunktionen verdeutlicht.

Zusammen mit anderen Disziplinen kann die Physiotherapie einen wichtigen Beitrag beim Management dieser Beschwerden leisten [9, 31]. Bei der physiotherapeutischen Behandlung von Kiefergelenkbeschwerden spielt die Manuelle Therapie eine wichtige Rolle [2, 23]. In diesem Zusammenhang wird in der Literatur vor allem die Manuelle Traktion nach kaudal mehrfach beschrieben $[2,15,19,23,26,27,36,39]$.

\section{Effektivität manueller Traktion}

Die Effektivität manueller Traktion kann auf verschiedene Weise erklärt werden. Bei der Betrachtung vom mechanistischen Modell 
her zeigt sie sich in einer Entlastung der Gelenkflächen, des Knorpels und des subchondralen Bereiches [14]. Neurophysiologische Denkmodelle erklären die Wirkung basierend auf Reflexmechanismen, bei denen periartikuläre Muskeln entspannt bzw. relaxiert werden $[10,11]$. Auch segmentale neurophysiologische Modulation lokal biologischer Effekte führt zu reduzierter Nozizeption und dadurch vermindertem Schmerz- und Muskeltonus [38].

Im Folgenden wird angenommen, dass die (Selbst-) Traktion mehr Raum zwischen den Gelenkpartnern schaffen soll und dadurch Schmerzen und intraartikuläre Dysfunktionen reduziert werden. Aufgrund der Mängel dieser Studie muss der Gedanke zurückgestellt werden, dass Separation einen Effekt auf Schmerzen und Funktion hat.

Obwohl aus klinischer Erfahrung Traktion in verschiedenen Fällen Schmerzen lindern und die Funktion verbessern kann [15, $31,39,37]$, sind aus Sicht der Autoren bislang keine Studien über die Effektivität einmaliger kurzzeitiger manueller Traktion des Kiefergelenks bekannt. Relevante Studien erforschten manuelle Therapie am Kiefergelenk als Langzeitbehandlung mit symptomlindernden Ergebnissen [2, 15, 19, 26, 27]. Aufgrund dieser Rechercheergebnisse entwickelte sich die vorliegende Studie mit der Untersuchung der Effektivität einmaliger kurzzeitiger manueller Traktion am Kiefergelenk von CMD-Patienten.

\section{Ultraschall am kraniomandibulären Gelenk}

Viele der gefundenen Studien zur Sonografie am kraniomandibulären Gelenk erforschten Ultraschall als diagnostisches Mittel zur Untersuchung der Genauigkeit, Sensitivität, Spezifität und Reliabilität [6-8, 12, 17, 25, 41]. Auch zur Distanz des Kiefergelenkspalts mittels Sonografie unter verschiedenen Gesichtspunkten finden sich in der Literatur Studien, jedoch mehr in Bezug auf die Darstellung einer indirekten Diskusposition, ohne jegliche Intervention [4].

Arbeiten zu Ultraschall am kraniomandibulären Gelenk beschäftigen sich meist mit der Fragestellung, ob er ein valides Mittel zur Diagnostik von Diskusverlagerungen ist [5-8, 17]. Hierbei wird oft die Übereinstimmung von Ultraschall und Magnetresonanztomografie (MRT) bei Diagnostik von CMD untersucht [6-8, 41]. Zur Untersuchung von Translationsbewegungen des Condylus mandibulae und Abständen des kraniomandibulären Gelenkspalts halten Landes und Sader [21] Ultraschall im Vergleich zum MRT (Übereinstimmung von $83 \%$ und 95\%) für ein genaues Instrument.

Diese vorherigen Studien zeigen, dass eine Visualisierung des kraniomandibulären Gelenkspalts mit Ultraschall möglich ist und zunehmend fokussiert wird.

\section{Fragestellung}

Die vorliegende Studie überprüfte die Hypothese, dass eine einmalige kurzzeitige manuelle Traktion einen Einfluss auf die Weite des Gelenkspalts des kraniomandibulären Gelenks bei CMD-Patienten hat und vergleicht diese Intervention mit einer Placebobehandlung. Dazu wurde Ultraschall als bildgebendes Verfahren eingesetzt und zudem die aktuelle Schmerzintensität untersucht.

\section{Methode}

Diese randomisierte kontrollierte Studie fand von September bis Oktober 2010 zum Teil in einer Zahnarztpraxis und in einer Physiotherapiepraxis an der Hochschule in Osnabrück statt.

Die Einschlusskriterien umfassten die von einem Zahnarzt auf den Kriterien der Research Diagnostic Criteria Temporomandibular Dysfunction (RDC/TMD) basierende diagnostizierte CMD. Zudem mussten ein Alter zwischen 20 und 70 Jahren und die Teilnahme auf freiwilliger Basis gegeben sein. Ausschlusskriterien waren eine Entzündung im Kiefergelenk sowie Operationen im Kiefergelenkbereich.

Alle Teilnehmer waren Patienten der oben erwähnten Zahnarztpraxis. 22 Probanden erfüllten die Voraussetzungen und wurden randomisiert einer Interventions- (IG) und Placebogruppe (PG) zugeteilt. Hierbei wurden die Lose von einer 2. Therapeutin zur Hälfte mit „I“ für Intervention und „P“ für Placebo beschriftet und vor der Behandlung blind gezogen. Die Untersuchungen und die Behandlung führte eine nicht verblindete Untersucherin durch, wohingegen alle Probanden während der gesamten Studie verblindet waren.

Sowohl Autorin als auch Therapeutin sind Physiotherapeutinnen mit 2 Jahren Berufserfahrung in der Behandlung muskuloskelettaler Erkrankungen. Beide absolvierten eine interne Fortbildung von 4 Stunden in Manueller Therapie auf dem Gebiet der kraniomandibulären Region durch einen klinischen Experten.

Den Probanden wurde erläutert, dass die Teilnahme freiwillig und der Rücktritt ohne Angaben von Gründen jederzeit und ohne irgendwelche Nachteile möglich seien. Sie wurden über die Randomisierung in die beiden Gruppen aufgeklärt. Ihnen wurde zugesichert, dass im Fall deutlich abweichender Behandlungserfolge die benachteiligte Gruppe nach Studienablauf mit der besseren Intervention nachbehandelt werden würde. Damit erfüllte die Untersuchung die ethischen Kriterien der Declaration of Helsinki der World Medical Association [42]. Aufgrund des Pilotstudiencharakters und der Einbettung durch andere Studien an gleichen Patientengruppen fand keine Powerberechnung statt.

\section{Assessments}

Die Veränderung der Gelenkspaltgröße wurde mit dem Ultraschallgerät Venue 40 von GE Healthcare untersucht. Dieses besteht aus einem Transducer (Schallkopf, der kurze Schallimpulse durch die zu untersuchende Körperregion gibt, die verschiedene Gewebe durch Echos reflektieren), einem Computer (Transmitter, der als Empfänger der Echos agiert und sie weiter zum Display sendet) und einem Visualisierungssystem (das Ultraschallbild anzeigendes Display) mit Speichergerät. Das Gerät erreicht eine Frequenz von bis zu $12 \mathrm{MHz}$, was für die Diagnostik bzw. Darstellung des Gelenks entscheidend ist [20].

Der Schallkopf wurde am liegenden Probanden longitudinal parallel zum Ohr angelegt und gekippt, bis sich ein optima-

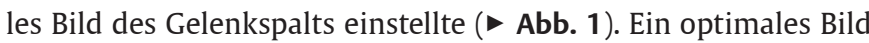
war gegeben, wenn die Knochenränder von Condylus mandibulae und Fossa articularis deutlich hyperechogen zu sehen waren. Eine Struktur wird als echogen bezeichnet, wenn sie die Fähigkeit hat, Schallwellen zu reflektieren. Hyperechogene Strukturen 
sind im Ultraschall weiß und hoch reflektierend dargestellt, wie z.B. Knochen, Pleura oder Muskelfaszien [16]. In dem Fall wurde das Bild „eingefroren“ und die Distanz des Gelenkspalts gemessen. Mithilfe eines Touchscreen-Stifts wurden auf dem Display 4 Distanzpunkte jeweils am visualisierten Knochenrand der Fossa articularis und des Condylus mandibulae gesetzt, der Abstand dazwischen vom US-Gerät berechnet und dessen Durchschnittswert ermittelt ( Abb. 2).

Zur subjektiven Einschätzung der momentanen Intensität des Schmerzempfindens diente die Coloured Analogue Scale (CAS) der Cranio Facial Therapy Academy (CRAFTA; Abb. 3). Diese farbige visuelle Analogskala (VAS) gilt als hoch reliables Messinstrument zur Bestimmung der subjektiven Schmerzintensität [22, 28, 32]. Die Probanden schoben die Markierung (schwarzer Strich) in den ihrem Schmerz entsprechenden Bereich, und die Therapeutin las den Wert auf der anderen Seite der Skala ab.

Da die Probanden - aus unbekannten Gründen - Schwierigkeiten bei der Einschätzung der genauen Schmerzintensität zu den verschiedenen Messmomenten (M1-M3) hatten, beschlossen die Autoren, statt einer numerischen eine ordinal skalierte Einteilung zu benutzen.

\section{Behandlung}

Ausgangsstellung, Griff und Dauer (4 Minuten) beider Behandlungen waren identisch ( Abb. 4). Die Interventionsgruppe erhielt eine einmalige longitudinale Bewegung nach kaudal (Traktion) im Grad IV nach Maitland. Abwechselnd wurden repetierend für ca. 10 Sekunden 1-2 Oszillationen pro Sekunde und eine anhaltende Technik am Bewegungslimit durchgeführt, ohne einen Schmerz über 3 (auf der Numeric Rating Scale) auszulösen.

Die einmalige Placebobehandlung unterschied sich lediglich in der Bewegungsdurchführung, bei der durch Zusammendrücken der kaudalen Hand ein Druck auf die Mandibula aufgebaut wurde. Mit dem gleichen Rhythmus wie bei der Intervention fanden oszillierende und anhaltende Bewegungen im Wechsel statt, ohne eine longitudinale Bewegung nach kaudal (Traktion) zu applizieren.

\section{Statistik}

Alle aufgenommenen Daten wurden bei SPSS 17 eingegeben und ausgewertet. Die Irrtumswahrscheinlichkeit (Signifikanzniveau) galt in Anlehnung an Bühl [1] bei $p \leq 0,05$ als signifikant, $p \leq 0,01$ als sehr signifikant, $\mathrm{p} \leq 0,001$ als höchst signifikant und $\mathrm{p}>0,05$ als nicht signifikant.

Zur Analyse der Vergleichbarkeit der einzelnen Probanden als Baseline-Messung wurde mithilfe des Chi-Quadrat-Tests nach Pearson geprüft, ob die Ausgangsdaten wie Alter und Geschlecht normalverteilt waren.

Zum Test eines signifikanten Unterschieds innerhalb der Gruppe diente der t-Test für verbundene Stichproben, da es sich bei den Werten der Distanzmessung um normalverteilte, intervallskalierte Daten handelte. Bei der CAS wurden die Daten als ordinalskalierte Daten interpretiert. Hierbei erfolgte die Berechnung der Unterschiede innerhalb der Gruppe anhand des beim Vergleich zweier abhängiger Stichproben mit Ordinalskalenniveau empfohlenen Rangsummentests nach Wilcoxon [1].

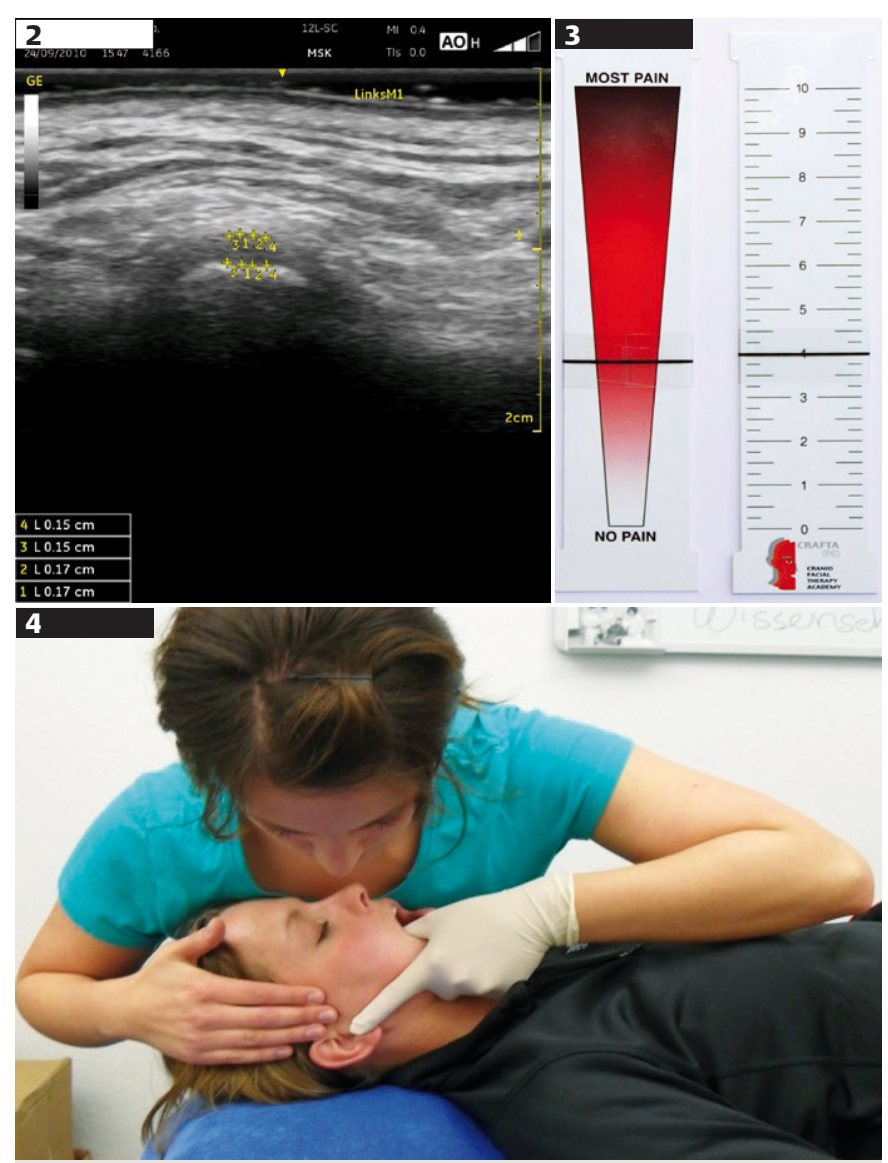

Abb. 2 Visualisierter Gelenkspalt des kraniomandibulären Gelenks im Ultraschallbild mit Distanzmesspunkten.

Abb. 3 Coloured Analogue Scale (CAS) zur Erhebung der momentanen Schmerzintensität.

Abb. 4 Ausgangsstellung und Griff der Interventions- sowie Placebobehandlung. Der Daumen der kaudalen Therapeutenhand liegt auf den kontralateralen unteren Backenzähnen der Probandin, die die Aufgabe hat, den Unterkiefer lockerzulassen.

Um festzustellen, ob ein signifikanter Unterschied der Distanzveränderungen zwischen beiden Gruppen bestand, wurde der tTest für unabhängige Stichproben verwendet [1]. Zur Signifikanzprüfung des Unterschieds zwischen den Gruppen bezüglich der jeweiligen Veränderung der CAS kam der Mann-Whitney-U-Test zum Einsatz, der laut Bühl [1] für 2 unabhängige ordinalskalierte Stichproben verwendet werden kann.

\section{Ergebnisse}

\section{Zusammensetzung der Stichprobe}

22 Probanden erfüllten die Einschlusskriterien und nahmen an der 1. Messung (M1) teil ( Tab. 1). Per Losverfahren wurden diese randomisiert einer Interventions- (IG) und einer Placebogruppe (PG) zugeteilt. Aufgrund unzureichender Darstellung des Condylus mandibulae und der Fossa articularis beiderseits fiel ein männlicher Teilnehmer der PG aus der Studie. Somit fanden die weiteren Untersuchungen und die Interventionen mit einer Stichprobe von $\mathrm{n}=21$ (IG: $\mathrm{n}=11$; PG: $\mathrm{n}=10$ ) statt. 


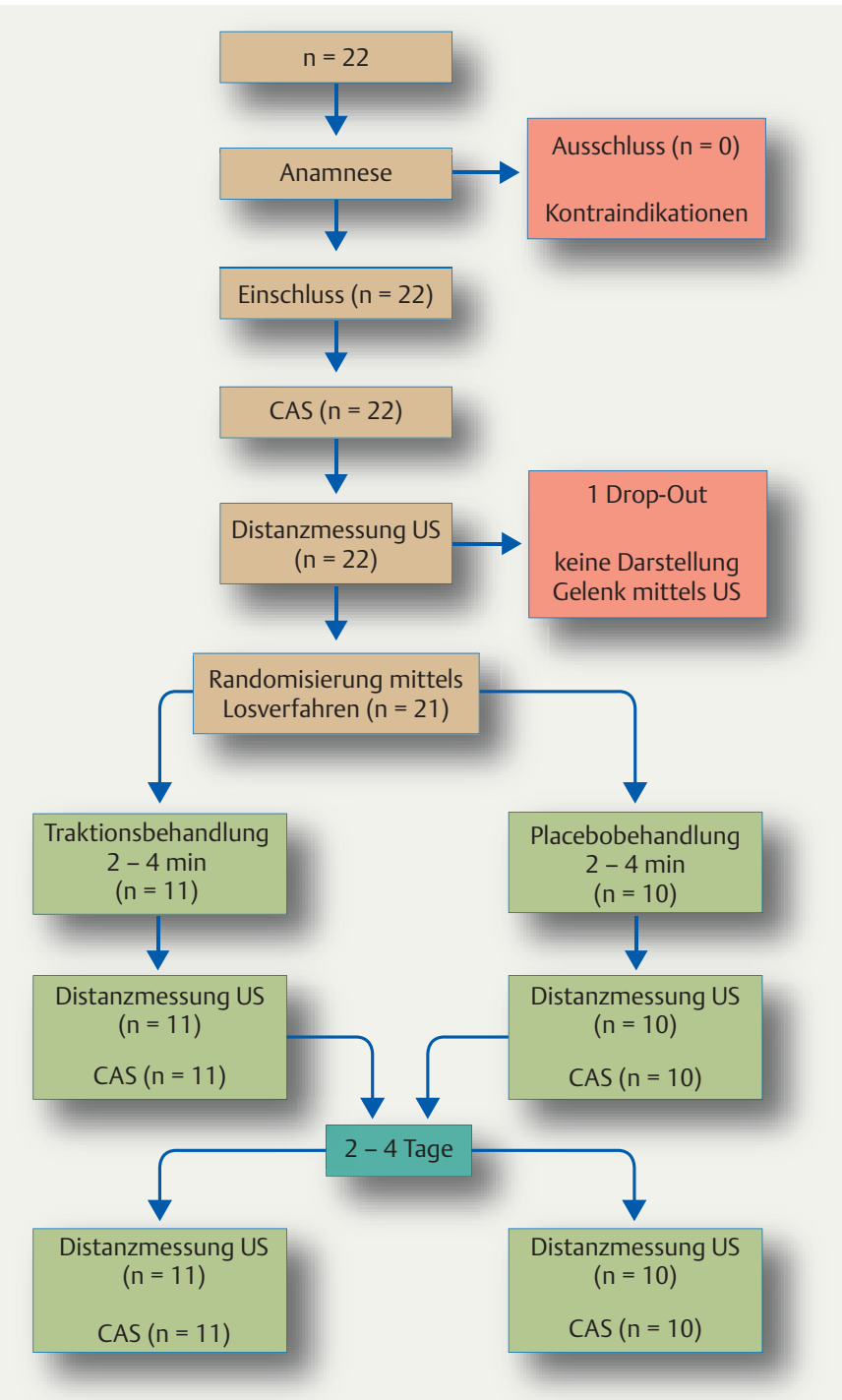

Abb. 5 Schematische Darstellung des Studienverlaufs (CAS = Coloured Analogue Scale; US = Ultraschall).

Zudem konnten aufgrund technischer Fehler des Venue 40 Ultraschallaufnahmen einer Probandin der IG nicht in einem leserlichen Format abgespeichert werden und fielen folglich aus der statistischen Auswertung. Hier wurde mit einer Stichprobengröße von $n=10$ der IG für die Auswertung der Distanzmessung fortgefahren. Die CAS wurde bei der Probandin wie bei allen Teilnehmern identisch abgefragt und die Behandlung durchgeführt ( Abb. 5).

\section{Statistische Auswertung}

\section{Distanzmessung}

Die Mittelwerte der IG stiegen durchschnittlich leicht an (durchschnittlicher Mittelwert: M1 = 0,0867 cm; M2 =0,0938 cm; M3 = 0,0978 cm; Abb. 6). Es ergab sich eine statistisch signifikante Vergrößerung des kraniomandibulären Gelenkspalts im Vergleich der Distanzwerte von M1 zu M3 mit p = 0,046* ( Tab. 3). Die PG wies im Durchschnitt ohne einen signifikanten Unterschied
Tab. 1 Daten der Drop-out-, nicht behandelten Probanden.

\begin{tabular}{|l|l|l|}
\hline & Geschlecht & Alter \\
\hline $\begin{array}{l}\text { Interventionsgruppe } \\
(\mathrm{n}=11)\end{array}$ & $\begin{array}{l}\mathrm{w}=9(82 \%) \\
\mathrm{m}=2(18 \%)\end{array}$ & $\begin{array}{l}\emptyset \text { 48 Jahre (SD } \pm 14,862) \\
\text { Minimum: 25 Jahre; } \\
\text { Maximum: 70 Jahre }\end{array}$ \\
\hline $\begin{array}{l}\text { Placebogruppe } \\
(\mathrm{n}=11)\end{array}$ & $\begin{array}{l}\mathrm{w}=7(70 \%) \\
\mathrm{m}=4(40 \%)\end{array}$ & $\begin{array}{l}\emptyset \text { 48 Jahre (SD } \pm 14,976) \\
\text { Minimum: } 20 \text { Jahre; } \\
\text { Maximum: 70 Jahre }\end{array}$ \\
\hline gesamt $(\mathrm{n}=22)$ & $\begin{array}{l}\mathrm{w}=16(72 \%) \\
\mathrm{m}=6(28 \%)\end{array}$ & $\begin{array}{l}\emptyset \text { 48 Jahre (SD } \pm 14,560) \\
\text { Minimum: 20 Jahre; } \\
\text { Maximum: 70 Jahre }\end{array}$ \\
\hline
\end{tabular}

$m=$ männlich; $n=$ Anzahl; $S D$ = Standardabweichung; $w$ = weiblich

Tab. 2 Distanzwerte vor, direkt nach und 2-4 Tage nach der Intervention und Placebobehandlung.

\begin{tabular}{|c|c|c|c|}
\hline & Distanz M1 & Distanz M2 & Distanz M3 \\
\hline \multicolumn{4}{|c|}{ Interventionsgruppe } \\
\hline $\begin{array}{l}\text { Mittelwert } \\
\text { Anzahl } \\
\text { SD } \\
\text { Median } \\
\text { Minimum } \\
\text { Maximum }\end{array}$ & $\begin{array}{l}0,086750 \mathrm{~cm} \\
10 \\
0,0309132 \mathrm{~cm} \\
0,087500 \mathrm{~cm} \\
0,0450 \mathrm{~cm} \\
0,1375 \mathrm{~cm}\end{array}$ & $\begin{array}{l}0,093750 \mathrm{~cm} \\
10 \\
0,0274684 \mathrm{~cm} \\
0,098750 \mathrm{~cm} \\
0,0525 \mathrm{~cm} \\
0,1500 \mathrm{~cm}\end{array}$ & $\begin{array}{l}0,097750 \mathrm{~cm} \\
10 \\
0,0289504 \mathrm{~cm} \\
0,095000 \mathrm{~cm} \\
0,0650 \mathrm{~cm} \\
0,1625 \mathrm{~cm}\end{array}$ \\
\hline \multicolumn{4}{|c|}{ Placebogruppe } \\
\hline $\begin{array}{l}\text { Mittelwert } \\
\text { Anzahl } \\
\text { SD } \\
\text { Median } \\
\text { Minimum } \\
\text { Maximum }\end{array}$ & $\begin{array}{l}0,113500 \mathrm{~cm} \\
10 \\
0,0350040 \mathrm{~cm} \\
0,108750 \mathrm{~cm} \\
0,0550 \mathrm{~cm} \\
0,1675 \mathrm{~cm}\end{array}$ & $\begin{array}{l}0,111000 \mathrm{~cm} \\
10 \\
0,0290402 \mathrm{~cm} \\
0,111250 \mathrm{~cm} \\
0,0625 \mathrm{~cm} \\
0,1500 \mathrm{~cm}\end{array}$ & $\begin{array}{l}0,112500 \mathrm{~cm} \\
10 \\
0,0389622 \mathrm{~cm} \\
0,120000 \mathrm{~cm} \\
0,0325 \mathrm{~cm} \\
0,1700 \mathrm{~cm}\end{array}$ \\
\hline
\end{tabular}

M1 = vor der Behandlung; M2 = direkt nach der Behandlung;

M3 = 2-4 Tage nach der Behandlung

eine Verkleinerung mit anschließender Vergrößerung des Mittelwerts auf (durchschnittlicher Mittelwert: M1 $=0,1135 \mathrm{~cm}$; M2 = $0,1110 \mathrm{~cm} ; \mathrm{M} 3=0,1125 \mathrm{~cm}$ ). Der Vergleich beider Gruppen zeigte keine signifikanten Differenzen (M1 zu M2: p = 0,264; M2 zu M3: $\mathrm{p}=0,831 ;$ M1 zu M3: $\mathrm{p}=0,304)$.

\section{CAS}

Die Mittelwerte der CAS wiesen bei der IG durchschnittlich eine Vergrößerung (durchschnittlicher Mittelwert: M1 = 0,91; M2 = 0,$95 ; \mathrm{M} 3=1,23$ ), bei der PG eine Reduzierung der Schmerzen auf (durchschnittlicher Mittelwert: M1 = 1,5; M2 = 1,23; M3 = 1,28; - Tab. 4, - Abb. 7). Der Vergleich beider Gruppen ergab keine signifikanten Unterschiede der CAS-Werte $(\bullet$ Tab. 5, $\bullet$ Abb. 7).

\section{Diskussion}

\section{Aktuelle Studienlage}

In Studien zur Wirkung Manueller Therapie bei CMD scheint sich die manuelle longitudinale Bewegung nach kaudal (Traktion) wie ein roter Faden durch die Behandlung von Zeichen und Symptomen 


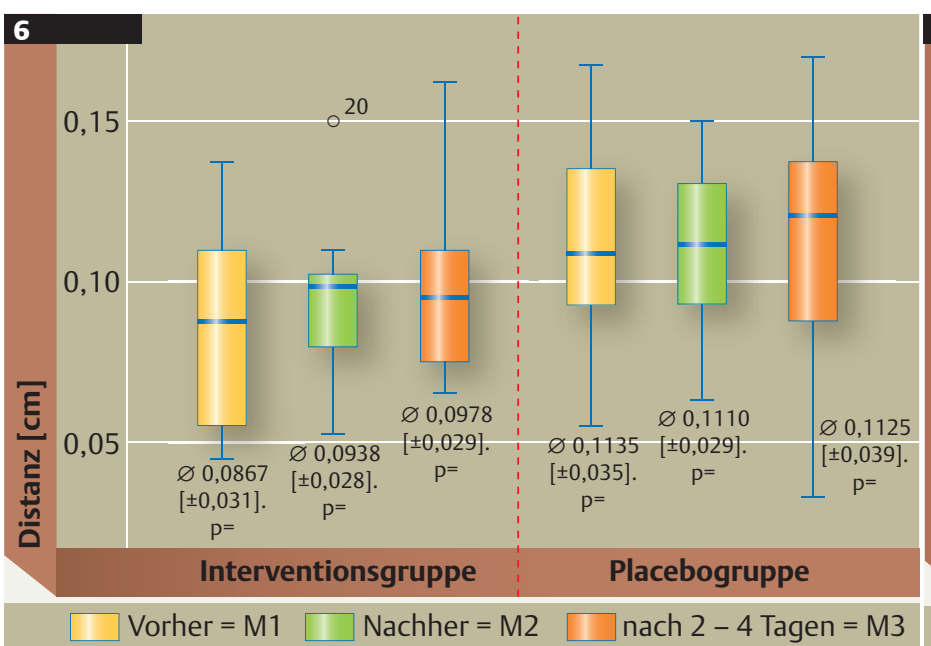

Abb. 6 Verhältnis der Gelenkspaltdistanz beider Gruppen vor (M1), direkt nach (M2) und 2-4 Tage nach der Behandlung (M3). Signifikanzwerte (p) M1 zu M2 (A), M2 zu M3 (B), M1 zu M3 (C); IG: $\mathrm{P}(\mathrm{A})=$ $0,143, p(B)=0,535, p(C)=0,046^{*} ; P G: p(A)=0,729, p(B)=0,881$, $\mathrm{p}(\mathrm{C})=0,925$.

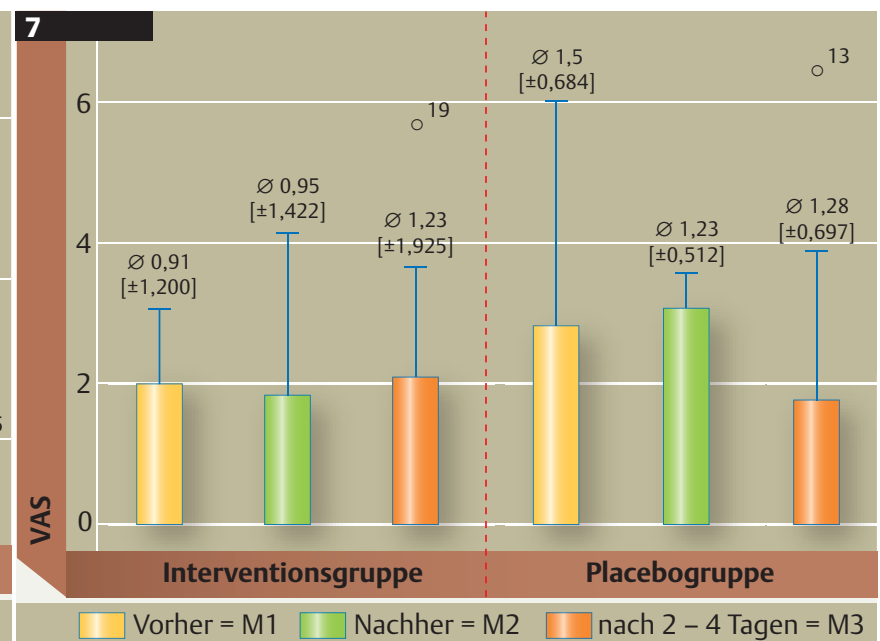

Abb. 7 Verhältnis der CAS-Mittelwerte beider Gruppen vor (M1), direkt nach (M2) und 2-4 Tage nach der Behandlung (M3). Signifikanzwerte (p) M1 zu M2 (A), M2 zu M3 (B), M1 zu M3 (C): IG: $P(A)=$ $0,705, p(B)=0,179, p(C)=0,465 ; P G: p(A)=0,285, p(B)=0,893, p(C)$ $=0,684$.
Tab. 3 Signifikanzwerte (p) der Distanzmessungen zum Vorher-nachher-Vergleich, 2-4 Tage nach der Behandlung und von der 1. bis zur letzten Messung innerhalb einer Gruppe und im Vergleich beider Gruppen.

\begin{tabular}{|l|c|c|c|}
\hline Distanz & M1 zu M2 & M2 zu M3 & M1 zu M3 \\
\hline Interventionsgruppe & $\mathrm{p}=0,143$ & $\mathrm{p}=0,535$ & $\mathrm{p}=0,046^{*}$ \\
\hline Placebogruppe & $\mathrm{p}=0,729$ & $\mathrm{p}=0,881$ & $\mathrm{p}=0,925$ \\
\hline $\begin{array}{l}\text { Vergleich Interventions- } \\
\text { und Placebogruppe }\end{array}$ & $\mathrm{p}=0,264$ & $\mathrm{p}=0,831$ & $\mathrm{p}=0,304$ \\
\hline
\end{tabular}

* = signifikant; (M1 = vor der Behandlung; $M 2$ = direkt nach der Behandlung; M3 = 2-4 Tage nach der Behandlung

in Gelenkstrukturen wie auch die der kraniomandibulären Region zu ziehen $[2,15,26,27,39]$. Trotz geringer Evidenz beschreiben Fachbücher die Wirkungsweise der Traktion allgemein und spezifisch für das kraniomandibuläre Gelenk und empfehlen die manuelle Traktion als Behandlungsmaßnahme [13, 14, 31, 34, 35].

Die Ergebnisse der vorliegenden Studie bestätigen nur teilweise diese Vorgaben. Zwar stellte sich in der IG nach 2-4 Tagen eine signifikante Vergrößerung des Gelenkspalts heraus ( $\mathrm{p}=0,046$; $\mathrm{n}$ $=10$ ), trotzdem ergab sich keine statistisch signifikante Verbesserung der Schmerzen (CAS: $\mathrm{p}=0,465$ ).

Die Autoren relevanter Studien [5-8, 12, 17, 25, 41] kommen zu dem Schluss, dass Ultraschall ein gutes Mittel zur Visualisierung des kraniomandibulären Gelenks darstellt, was die vorliegende Arbeit bestätigt.

\section{Veränderungen der Untersuchungsparameter}

Die Ergebnisse wurden möglicherweise durch die Tatsache beeinflusst, dass die Untersuchungen der Probanden teilweise an verschiedenen Studienorten, Tageszeiten sowie Wochentagen statt-
Tab. 4 Coloured Analogue Scale (CAS) vor, direkt nach und 2-4 Tage nach der Behandlung.

\begin{tabular}{|l|l|l|l|}
\hline & CAS M1 & CAS M2 & CAS M3 \\
\hline Interventionsgruppe & & & \\
\hline Mittelwert & 0,9091 & 0,9545 & 1,2273 \\
Anzahl & 11 & 11 & 11 \\
Standardabweichung & 1,20038 & 1,42223 & 1,92501 \\
Median & 0,0000 & 0,0000 & 0,0000 \\
Minimum & 0,00 & 0,00 & 0,00 \\
Maximum & 3,00 & 4,25 & 5,50 \\
\hline Placebogruppe & & & \\
\hline Mittelwert & & & \\
Anzahl & 1,5000 & 1,2250 & 1,2750 \\
Standardabweichung & 10 & 10 & 10 \\
Median & 2,16346 & 1,61783 & 2,20306 \\
Minimum & 0,0000 & 0,0000 & 0,0000 \\
Maximum & 0,00 & 0,00 & 0,00 \\
\hline & 6,00 & 3,50 & 6,50 \\
\hline
\end{tabular}

- Tab. 5 Signifikanzwerte (p) des Vergleichs beider Gruppen in Bezug auf Schmerz.

\begin{tabular}{|l|l|l|l|}
\hline & M1 zu M2 & M2 zu M3 & M1 zu M3 \\
\hline CAS & $\mathrm{p}=0,966$ & $\mathrm{p}=0,845$ & $\mathrm{p}=0,558$ \\
\hline
\end{tabular}

CAS = Coloured Analogue Scale; $M 1$ = vor der Behandlung; $M 2$ = direkt nach der Behandlung; M3 = 2-4 Tage nach der Behandlung

fanden. Zudem sind unterschiedliche Bedingungen zu berücksichtigen, wie Lichtverhältnisse und somit die Ultraschallbildqualität sowie die Behandlungsbank.

Auch der Einfluss biopsychosozialer Faktoren ist zu beachten. Sympathie als auch Antipathie zwischen Therapeutin und Proban- 
den, Erfahrung mit (intraoraler) Physiotherapie und Schmerzen oder anderen Beschwerden spielen bei der Therapiewirkung eine Rolle.

Die Ergebnisse in der PG lassen vermuten, dass das Zusammendrücken der Mandibula und von Weichteilen des Kausystems einen arthrokinematischen, neurophysiologischen Einfluss haben könnte. Aufgrund der klinisch signifikanten Vergrößerung des Gelenkspalts in der IG und keiner signifikanten Veränderung der subjektiven Angaben der Probanden der IG steht die Vergrößerung des Gelenkspalts vermutlich nicht in Zusammenhang mit einer subjektiven Antwort der Patientengruppe. Zudem stellt sich auch die Frage, ob die statistische Signifikanz der Gelenkspaltvergrößerung auch tatsächlich klinisch signifikant ist. Laut Autoren ist in der Literatur kein Smallest detectable change (kleinster zu beobachtender Unterschied) bekannt.

Die Schmerzintensität zeigte 2-4 Tage nach der Intervention ebenso wie nach der Placebobehandlung keine signifikanten Veränderungen auf. Dies weist daraufhin, dass Gelenkspaltvergrößerung nicht direkt mit Schmerzlinderung korreliert. Es stellt sich die Frage, ob eine Traktion, wie sie in dieser Studie durchgeführt wurde, überhaupt eine Maßnahme zur Schmerzlinderung ist. Möglicherweise sind die Dauer der Behandlung und die Tatsache, dass es sich lediglich um eine einmalige Applikation handelte, entscheidende Faktoren. Aber auch die unterschiedlichen Ausgangsdaten (durchschnittlicher Mittelwert) zu Beginn der Messungen im Vergleich beider Gruppen sind zu berücksichtigen. Ein erhöhtes Verbesserungspotenzial der Schmerzen der PG kann vermutet werden ( $\bullet$ Tab. 4).

\section{Methode}

Aufgrund der kleinen Stichprobe von 22 Teilnehmern ist eine Generalisierbarkeit der Ergebnisse nicht möglich. Eine Vergleichbarkeit der soziodemografischen Daten innerhalb der IG und der PG sowie zwischen beiden Gruppen war hier jedoch gegeben und eine Randomisierung durchführbar.

Zur Visualisierung eignet sich die Anwendung von Ultraschall, jedoch gab es auch Nachteile. Das Verwackeln von Ultraschallbildern durch die Bedienung nur eines Therapeuten sowie eine standardisierte Schallkopfanlage bei mehrfacher Kippung für ein optimales Bild waren teilweise problematisch. Eine mögliche Verzerrung der Ergebnisse könnte außerdem durch die nicht verblindete Therapeutin entstanden sein.

\section{Schlussfolgerungen}

In der vorliegenden Studie erfolgte eine 4-minütige einmalige kurzzeitige manuelle Traktion des kraniomandibulären Gelenks im Vergleich zu einer Placebobehandlung bei 2 homogenen Gruppen. Dabei zeigten sich direkt nach der Behandlung in beiden Gruppen keine signifikanten Veränderungen der Gelenkspaltdistanz. Erst 2-4 Tage nach der Applikation scheint die Traktion im Vergleich zur Ausgangsmessung innerhalb der IG eine signifikante Distanzvergrößerung des Gelenkspalts zu bewirken. Die Placebobehandlung ergab keine signifikanten Veränderungen des Gelenkspalts in der PG.

Bezüglich des Parameters Schmerz scheinen die Applikationen sowohl auf die IG als auch auf die PG keinen signifikanten Einfluss zu haben.

\section{Ausblick}

Für eine deutlichere Aussage über die Veränderung des Gelenkspalts durch Manuelle Therapie ebenso wie anderer Interventionen (z. B. Übungs- und Schienentherapie) bedarf es einer größeren Stichprobe und feinerer Einschlusskriterien, um die Ergebnisse zu verallgemeinern. Eine doppelte Verblindung und wiederholtes Messen durch eine oder mehrere Therapeuten reduzieren die Gefahr möglicher Messfehler und Ergebnisverzerrungen.

Um die Effektivität von Traktion zu messen, sind zudem noch weitere Parameter und Assessments erforderlich, wobei Ultraschall sicherlich eine wichtige Rolle spielt. Vor allem für Funktionsaufnahmen des Kiefergelenks bietet Ultraschall zwar einen großen Vorteil, jedoch ist die Technik nicht ausgereift. Die optimale Differenzierung der Strukturen erfordert eine langjährige Erfahrung.

Die Studie konnte aufzeigen, dass Ultraschall ein unterstützendes Messinstrument zur Beurteilung des Gelenkspalts bei kraniomandibulären Untersuchungen, Behandlungen und Reassessments darstellt. Aufgrund des Mangels an weiteren Studien zu Ultraschall in der kraniomandibulären Region liegen keine Referenzwerte vor. Dennoch ist Ultraschall ein wertvolles Messinstrument unter anderem bei intraartikulären kraniomandibulären Dysfunktionen, um die klinische Entscheidung des Manualtherapeuten zu vereinfachen und zu verbessern.

Mehr Evidenz anhand von Untersuchungen der Effektivität manueller Traktion mit reduzierten Fehlerquellen ist für die $\mathrm{Zu}$ kunft wünschenswert.

\section{Danksagung}

Wir danken der Fa. GE Healthcare für die freundliche Unterstützung bei der Durchführung der Ultraschalluntersuchungen und die Zurverfügungstellung des Ultraschallgeräts Venue 40.

\section{Literatur}

1. Bühl A. SPSS 14. Einführung in die moderne Datenanalyse. München: Pearson, 2006

2. Carmeli E, Sheklow SL, Bloomenfeld I. Comparative Study of Repositioning Splint Therapy and Passive Manual Range of Motion Techniques for Anterior Displaced Temporomandibular Discs with Unstable Excursive Reduction. Physiotherapy 2000; 87: 26-36

3. Cuccia AM, Caradonna C, Annunziata V, Caradonna D. Osteopathic manual therapy versus conventional conservative therapy in the treatment of temporomandibular disorders: a randomized controlled trial. Journal of bodywork and movement therapies 2010; 14: 179-184

4. Elias FM, Birman EG, Matsuda CK, de Souza Oliveira IR, Jorge WA. Ultrasonographic findings in normal temporomandibular joints. Braz Oral Es 2006; 20: 25-32

5. Emshoff R, Bertram S, Rudisch A, Gaßner R. The diagnostic value of ultrasonography to determine the temporomandibular joint disc position. Oral Surgery Oral Medicine Oral Pathology 1997; 84: 688-696

6. Emshoff R, Jank S, Rudisch A, Bodner G. Are High-Resolution Ultrasonographic Signs of Disc Displacement Valid? Journal of Oral Maxillofacial Surgery 2002; 60: 623-628

7. Emshoff R, Jank S, Bertram S, Rudisch A, Bodner G. Disk Displacement of the Temporomandibular Joint: Sonography Versus MR Imaging. American Journal of Roentgenology 2002; 178: 1557-1562

8. Emshoff R, Jank S, Rudisch A, Walch C, Bodner G. Error patterns and observer variations in the high-resolution ultrasonography imaging evaluation of the disk position of the temporomandibular joint. Oral Surgery Oral Medicine Oral Pathology 2002; 93: 369-375 
9. Fink MG, Tschernitschek H, Schliephake H et al. Physikalische Medizin in der Zahnheilkunde - Klassifizierung der Myoarthropatien. Phys Rehab Kur Med. 2001; 11: 221-228

10. Geiringer SR, Kincaid CB. Rechtien JJ. Traction, manipulation, and massage. In: DeLisa JA (ed). Rehabilitation Medicine: Principles and Practice. Philadelphia, Lippincott, 1988

11. Harris R. Traction. In: Licht S (ed). Massage, Manipulation, and Traction. New York: Krieger, 1976

12. Hayashi T, Ito J, Koyama J, Yamada K. The Accuracy of Sonography for Evaluation of Internal Derangement of the Temporomandibular Joint in Asymptomatic Elementary School Children: Comparison with MR and CT. American Journal of Neuroradiology 2001; 22: 728-734

13. Hengeveld E, Banks K. Maitlands Manipulation der peripheren Gelenke. München: Elsevier Urban \& Fischer, 2008

14. Hüter-Becker A, Dölken M. Physiotherapie in der Orthopädie. Stuttgart: Thieme, 2005

15. Ismail F, Demling A, Heßling K, Fink M, Stiesch-Scholz M. Short-term efficacy of physical therapy compared to splint therapy in treatment of arthrogenous TMD. Journal of Oral Rehabilitation 2007; 34: 807-813

16. Ivani G, Mosetti V. Ultrasound and Regional Anesthesia. München: GE Healthcare, 2008

17. Jank S, Rudisch A, Bodner G et al. High-resolution ultrasonography of the TMJ: helpful diagnostic approach for patients with TMJ disorders? Journal of Cranio-Maxillofacial Surgery 2001; 29: 366-371

18. John MT, Zwijenburg AJ. Interobserver variability in assessment of signs of TMD. Int J Prosthodont 2001; 14: 265-270

19. Knust M, von Piekartz HJM, Zalpour C. Wirkung von Manueller Therapie im Vergleich zu einem multimodalen Physiotherapieprogramm bei Patientinnen mit kraniomandibulärer Dysfunktion. Pilotstudie. physioscience 2007; 3: 109-116

20. Köstering B, Jost-Köstering S, Dudwiesus H. Medical Technology and Medical Information Systems: Imaging Systems/Ultrasound Diagnostics. Medizintechnik. Berlin: Springer, 2001

21. Landes CA, Sader R. Sonographic evaluation of the ranges of condylar translation and of temporomandibular joint space as well as first comparison with symptomatic joints. Journal of Cranio-Maxillofacial Surgery 2007; 35: 374-381

22. McGrath PA, Seifert CE, Speechley KN et al. A new analogue scale for assessing children`s pain: an initial validation study. Pain 1996; 64: 435-443

23. McNeill C. Management of temporomandibular disorders: Concepts and controversies. The Journal of Prosthetic Dentistry 1997; 77: 511-522

24. Medlicott MS, Harris SR. A Systematic Review of the Effectiveness of Exercise, Manual Therapy, Electrotherapy, Relaxation Training, and Biofeedback in the Management of Temporomandibular Disorder. Physical Therapy 2006; 86: 955-973

25. Mohl ND. Reliability and Validity of Diagnostic Modalities for Temporomandibular Disorders. Advances in Dental Research 1993; 7: 113-119

26. Nicolakis P, Erdogmus B, Kopf A et al. Exercise Therapy for Craniomandibular Disorders. Arch Phsy Med Rehabil 2000; 81: 1137-1142

27. Nicolakis P, Erdogmus B, Kopf A et al. Effectiveness of exercise therapy in patients with myofascial pain dysfunction syndrome. Journal of Oral Rehabilitation 2002; 29: 362-368

28. Oesch P, Hilfiker R, Keller S et al. Assessments in der muskuloskelettalen Rehabilitation. Bern: Huber, 2007

29. Palla S. Grundsätzliches zur Diagnose der Myoarthropathien. In: Palla S (Hrsg). Myoarthropathien des Kausystems und orofaziale Schmerzen. Zürich: Klinik für Kaufunktionsstörungen und Totalprothetik, 1998

30. Peroz I. Otalgie und Tinnitus bei Patienten mit kraniomandibulären Dysfunktionen. HNO 2001; 49: 713-718

31. Von Piekartz HJM. Kiefer, Gesichts- und Zervikalregion. Neuromuskuloskeletale Untersuchung, Therapie und Management. Stuttgart: Thieme, 2005

32. Von Piekartz HJM, Lüdtke K. Effect of treatment of temporomandibular disorders (TMD) in patients with cervicogenic headache: a single-blind, randomized controlled study. Cranio 2011; 29: 43-56

33. Saunders HD. Use of spinal traction in the treatment of neck and back conditions. Clin Ortbop 1983; 179: 31-38
34. Schomacher J. Manuelle Therapie. Bewegen und spüren lernen. Stuttgart: Thieme, 2007

35. Schomacher J. Selbsttraktion des Kiefergelenks führt zur Separation zwischen Kondylus und Fovea articularis im CT. manuelletherapie 2011; 15: 105-108

36. Schupp W, Marx G. Manuelle Behandlung der Kiefergelenke zur Therapie der kraniomandibulären Dysfunktion. Manuelle Medizin 2002; 40: 177-183

37. Stelzenmüller W, Wiesner J. Therapie von Kiefergelenkschmerzen. Ein Behandlungskonzept für Zahnärzte, Kieferorthopäden und Physiotherapeuten. Stuttgart: Thieme, 2010

38. Swezey RL. The modern thrust of manipulation and traction therapy. Semin Arthritis Rheum 1983; 12: 322-331

39. Tanaka E, Detamore MS, Mercuri LG. Degenerative Disorders of the Temporomandibular Joint: Etiology, Diagnosis, and Treatment. Journal of Dental Research 2008; 87: 296-307

40. Tjakkes GHE, Reinders JJ, Tenvergert EM, Stegenga B. TMD pain: the effect on health related quality of life and the influence of pain duration. Health and Quality of Life Outcomes 2010; 8: 46. DOI: 10.1186/1477-7525-8-46

41. Uysal S, Kansu H, Akhan O, Kansu Ö. Comparison of ultrasonography with magnetic resonance imaging in the diagnosis of temporomandibular joint internal derangements: A preliminary investigation. Oral Surgery Oral Medicine Oral Pathology 2002; 94: 115-121

42. World Medical Association (WMA). Declaration of Helsinki. 2008. www. wma.net/en/30publications/10policies/b3

\section{AUTORIN E}

Anne-Marie Schmitz, BSc PT

Wörthstr. 25

49080 Osnabrück

anne.schmitz@kabelmail.de

\section{BIBLIOGRAFIE}

DOI 10.1055/s-0032-1304758

manuelletherapie 2012; 16: 34-41

(c) Georg Thieme Verlag KG

Stuttgart · New York · ISSN 1433-2671 